\title{
Editorial
}

\section{Medikamente: Spiegel der Problematik des medizinischen Fortschritts}

\author{
R. Bruppacher ${ }^{1}$
}

Während Tausenden von Jahren vertrauten die Menschen der Heilkraft von Arzneien. Rückblickend können wir feststellen, dass dieses Vertrauen bis vor kurzer Zeit meist mehr zur Heilung beigetragen hat als das Medikament selbst. In den letzten 40 Jahren hat die Pharmakotherapie einen gewaltigen Aufschwung genommen. Wir verfügen heute über eine Vielzahl von Medikamenten, deren Wirksamkeit sorgfältig nachgewiesen wurden. Sie unterliegen einer laufenden Kontrolle bezüglich Qualität und Sicherheit, welche das übliche Ausmass weit übertreffen.

Dennoch hat das Vertrauen in den Nutzen der Pharmakotherapie in weiten Kreisen mindestens teilweise einem Unbehagen und Misstrauen Platz gemacht. Mit der Wirksamkeit sind auch die Gefahren falscher Anwendung gestiegen. Wie immer, wenn sich die Potenz moderner Wissenschaft mit der Möglichkeit menschlichen Fehlverhaltens paart, entsteht das Gefühl der Bedrohung. Das Medikament steht häufig im Brennpunkt der Kritik am heutigen Gesundheitswesen. Diese wird von vielen, die sich mit ihrer ganzen Kraft und Überzeugung für die Bekämpfung menschlichen Leidens einsetzen, als ungerechtfertigt und schmerzlich empfunden. Sie richtet sich aber nicht gegen die guten Absichten der Verfechter des medizinischen Fortschrittes, sondern gegen die unbeabsichtigten Neben- und Sekundärwirkungen. Die Thalidomid-Tragödie hat einen bleibenden Eindruck hinterlassen. In Übereinstimmung mit dem traditionellen Leitsatz «Primum nil nocere» wurden staatliche Kontrollen eingerichtet, die Entwicklung, Vertrieb und Anwendung der Medikamente an immer steigende Auflagen knüpfen. Dies hat bewirkt, dass nur noch hoch spezialisierte Arbeitsgruppen Entwicklung, Herstellung und Verkauf von Präparaten mit einiger Aussicht auf Erfolg betreiben können. Die pharmazeutische Industrie ist so zu einem wichtigen Partner der Medizinalberufe geworden.

Der Fortschritt in der Medizin war aber auch mit einer massiven Erhöhung der Kosten für das Gesundheitswesen verbunden. Obwohl die Medikamente daran weit weniger beteiligt waren als andere Elemente der Behandlung und Pflege, richten sich Bemühungen um Kostendämpfung gerne auf dieses Ziel, das nicht direkt mit der persönlichen Betreuung verbunden, sondern eben industriell gefertigt ist. Die Úberwälzung der medizinischen Behandlungskosten an staatlich subventionierte Sozialversicherungen hat weitere Interessenten einer Kontrolle der Anwendung von Medikamenten auf den Plan gerufen.

Fragen der medizinischen Behandlung werden zunehmend in der Öffentlichkeit diskutiert. Informationen und «Anweisungen» hinsichtlich des Gebrauchs selbst rezeptpflichtiger Präparate erfolgen auch von nichtärztlicher Seite in der Laienpresse. Die traditionelle Rolle der Medizinalberufe ist in Frage gestellt.

Die Schweizerische Gesellschaft für Sozial- und Präventivmedizin in ihren Bemühungen, ein Forum für interdisziplinäre Gespräche als Ausgangspunkt sachbezogener Lösungen im Interesse unserer Bevölkerung zu bieten, stellte die traditionellerweise einem aktuellen Thema aus dem Gesundheitswesen gewidmete Herbsttagung unter den Titel «Das Medikament im heutigen Gesundheitswesen». Die in diesem Heft abgedruckten Referate und Berichte geben einen Eindruck von der lebhaften, aber konstruktiven Auseinandersetzung mit dieser konfliktträchtigen Materie. Als logischer Partner für die Veranstaltung diente der Schweizerische Apothekerverein und besonders der Ordine dei Farmacisti del Cantone Ticino, dessen Präsident, Herr Luigi Varini, als Leiter des Organisationskomitees fungierte. $\mathrm{Thm}$ und dem Tagungspräsidenten, Prof. Boris Luban-Plozza, wie allen Beteiligten gebührt herzlicher Dank für ihre Anstrengungèn, Brücken zwischen den Standpunkten zu schlagen und die Probleme um den Einsatz der Medikamente zu entschärfen.

1 PD Dr. med., Dr. P. H., Abteilung für Sozial- und Präventivmedizin der Universität Basel,

c/o Programmleitung NFP 8, Kantonsspital, CH-4031 Basel. 